\title{
SMOS-NEXT: A NEW CONCEPT FOR SOIL MOISTURE RETRIEVAL FROM PASSIVE INTERFEROMETRIC OBSERVATIONS
}

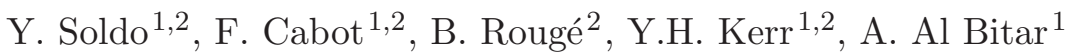 \\ and E. Epaillard ${ }^{1}$
}

\begin{abstract}
Present soil moisture and ocean salinity maps retrieved by remote sensing are characterized by a coarse spatial resolution. Hydrological, meteorological and climatological applications would benefit greatly from a better spatial resolution. Owing to the dimensions of the satellite structure and to the degradation of the instrument's radiometric sensitivity, such improvement cannot be achieved with classical interferometry. Then, in order to achieve this goal an original concept for passive interferometric measurements is described. This concept should allow to achieve a much finer spatial resolution, which can be further improved with the application of disaggregation methods. The results will then allow the integration of global soil moisture maps into hydrological models, a better management of water resources at small scales and an improvement in spatial precision for various applications.
\end{abstract}

\section{Introduction}

During the last decades the need for a global estimation with high temporal resolution of key environmental variables such as soil moisture and ocean salinity has grown greatly (Robock et al. 2000; Dai et al. 2004; Roemmich et al. 2000).

Satellites represent the best mean for satisfying such need, and several instruments have been launched onboard European and American satellites with the intent of retrieving large-scale soil moisture and ocean salinity maps.

These instruments are based on different principles. They may involve radiometers (Njoku et al. 2003), scatterometers (Bartalis et al. 2007), interferometric radiometers (Kerr et al. 2001), or they may rely on both passive and active elements (LeVine et al. 2007; Entekhabi et al. 2010).

\footnotetext{
${ }^{1}$ Centre d'Études Spatiales de la Biosphère (CESBIO), 18 avenue Edouard Belin, bpi 2801, 31401 Toulouse Cedex 9, France; e-mail: yan.soldo@cesbio.cnes.fr

2 Centre National d'Études Spatiales (CNES), 18 avenue Edouard Belin, 31401 Toulouse Cedex 9, France
} 


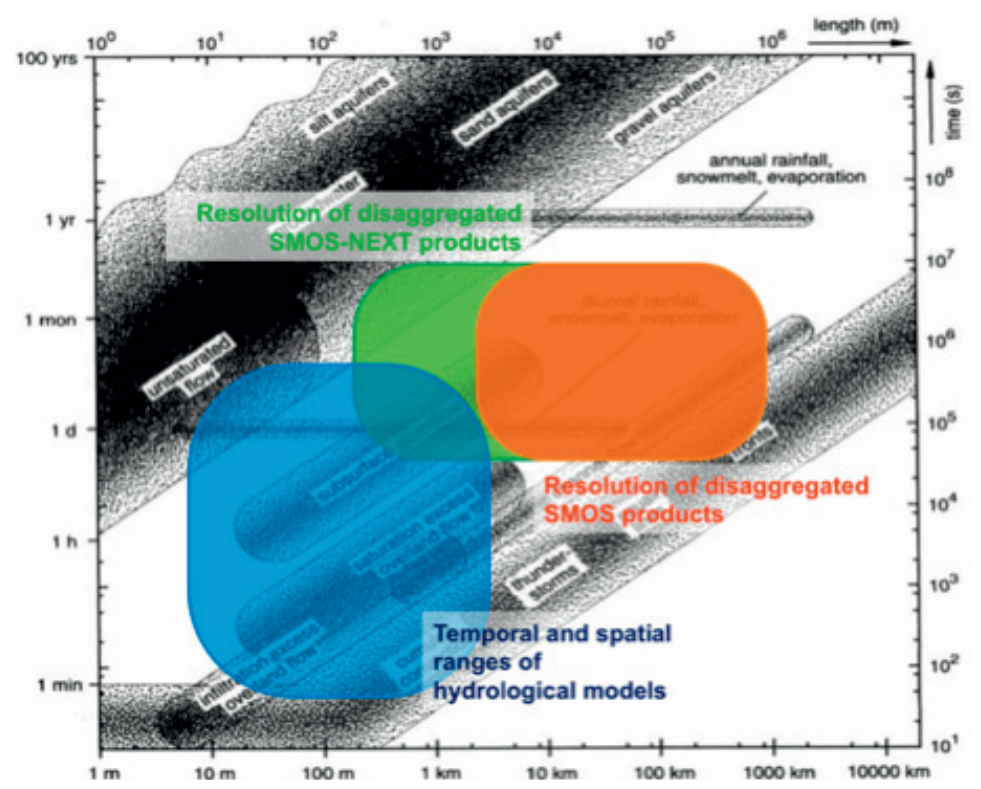

Fig. 1. Characterization of spatial and temporal scales for hydrological models and disaggregated remote sensed data.

Although different technologies were adopted, all these instruments are limited by a spatial resolution of few tens of kilometers.

In order to be able to make use of these data in hydrological models, and for many other applications, like the survey of water resources at the scale of irrigated zones, a better spatial resolution must be achieved, typically it should be improved by an order of magnitude.

To assure continuous monitoring of soil moisture and ocean salinity, while attaining an unprecedented fine spatial resolution, an original concept was proposed in Cabot et al. (2012) and Kerr et al. (2010), which aims at achieving a spatial resolution of few kilometers while maintaining roughly the same radiometric resolution.

\section{The need for high spatial resolution}

The management of water resources is already, and will be even more in the future, a critical issue (Alcamo et al. 2000; Döll et al. 2003). Properly dealing with this issue cannot be done without a deep understanding of the processes involved in the water cycle, which are studied using hydrological models.

Temporal and spatial scales can vary significantly from one model to the other depending on the processes they focus on. However, by improving the spatial resolution, the interaction between remotely sensed global soil moisture maps and hydrological models will be more effective and will result in an improved 
environmental knowledge. Figure 1 shows how disaggregation of SMOS-NEXT retrievals will result in a better cooperation with hydrological models.

Combined with weather models, remotely sensed global soil moisture maps can help achieve more accurate forecasting predictions, as well as to assess the risk for fires or floods on specific areas. Storms or heavy precipitations are of course more likely to cause floods over moist soils, and winds over very dry soils will increase the risk for fires.

Over ocean, the salt content will be retrieved. Indeed, ocean salinity's annual and inter-annual variations are crucial for monitoring and understanding of climate and climate changes, as they influence ocean currents and water evaporation from oceanic surfaces. A better resolution will improve the capability to follow in more details how currents vary with time as well as how river plumes interact with these oceanic currents.

Other than the nominal uses, SMOS has proved to be a versatile satellite, as its data has been used also for applications like wind speed estimation inside tornadoes (Grodsky et al. 2012) or the monitoring of the extent of sea ice sheets (Kaleschke et al. 2012). Naturally all these applications will benefit from a finer spatial resolution.

\section{Operating frequency and spatial resolution}

The maximum sensitivity to both soil moisture and ocean salinity is close to the protected 1400-1427 MHz band, and atmospheric disturbances are negligible at these frequencies (Wigneron et al. 2000), thus for a passive instrument like SMOS-NEXT, this wave band is clearly the best choice in term of operating frequency.

Even though artificial emissions are forbidden in this band to allow passive observations of both Earth and sky (ITU Radio Regulations 1996), after the first SMOS' data retrievals, the presence of contaminating unlawful sources was noticed (Anterrieu \& Khazaal 2011), so a strategy has been developed to deal with these radio frequency interferences that should provide a cleaner signal. The detailed description of this strategy is out of the scope of this contribution.

Once the operating frequency has been fixed, there are only two other parameters that define the spatial resolution $\left(R_{s}\right)$

$$
R_{s}=\frac{H \lambda}{d}
$$

$H$, the satellite altitude, and $d$, the diameter of the equivalent real aperture antenna, that in our case is equal to the maximum baseline ( $\lambda$ is the wavelength of the central operating frequency).

The spatial resolution could be improved by reducing the altitude of the satellite. However, the choice of altitude is also driven by constraints linked to the width of the swath and to the density of the atmosphere which determines the fuel consumption, and hence the weight at launch. All similar missions are orbiting, or are planned to orbit, at altitudes of about $700 \mathrm{~km}$, so it is safe to assume that a similar value will be chosen. 


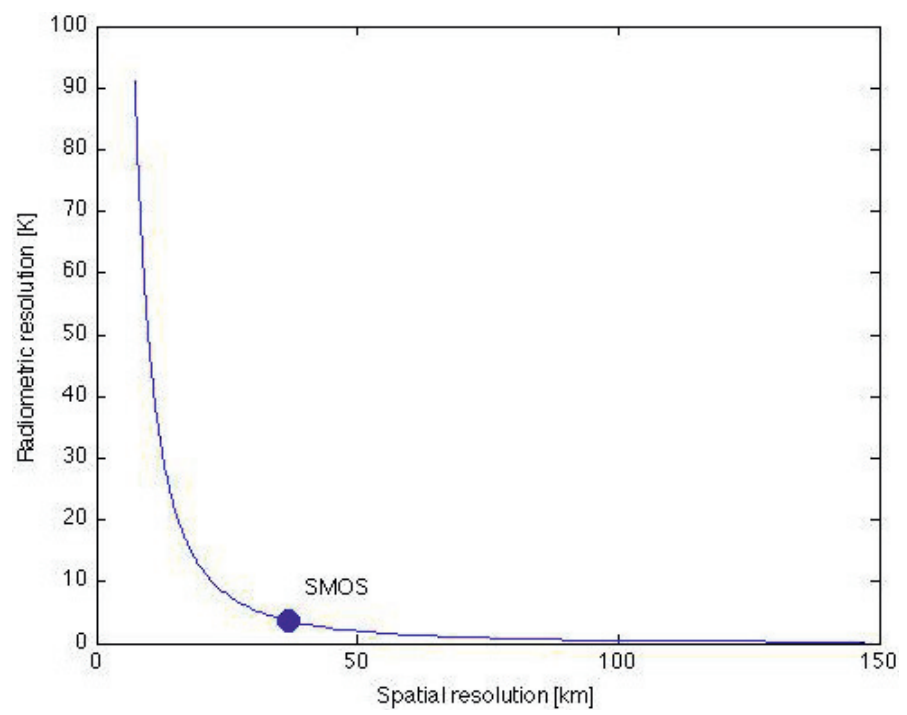

Fig. 2. Interdependence between spatial resolution and radiometric sensitivity; SMOS' maximal baseline is already close to the optimal trade off.

Consequently, the only way to improve the resolution is to increase the length of the baselines.

However, longer baselines means bigger surfaces of the equivalent real aperture antenna, and that is detrimental to the radiometric resolution $(\Delta T)$ (Camps et al. 1998) according to:

$$
\Delta T=A \frac{T_{A}+T_{r e c}}{\sqrt{B t_{i}}} \sqrt{N_{V}}
$$

where $A$ is the pixel area, $T_{A}$ is the antenna temperature, $T_{r e c}$ is the receiver temperature, $B$ is the spectral bandwidth, $t_{i}$ is the integration time interval and $N_{V}$ is the number of points sampled by the array in the Fourier domain.

To improve the spatial resolution by an order of magnitude means to have baselines ten times bigger. For SMOS this would lead to three $40 \mathrm{~m}$ long arms, which represents obvious feasibility difficulties. Moreover, as the spatial resolution vary linearly with $d$, the radiometric resolution is proportional to the square of $d$, through $N_{V}$ (for a Y-shaped instrument $N_{V}=6 N_{e l}^{2}+6 N_{e l}+1$, with $N_{e l}$ the number of receivers per arm); hence longer baselines would lead to a loss of radiometric sensitivity, which is unacceptable with respect to the very stringent requirements for oceanic observations (Berger et al. 2002).

Because of this relation between spatial resolution and radiometric sensitivity, this classical approach can hardly lead to the improvement of one without the degradation of the other (see Fig. 2). 


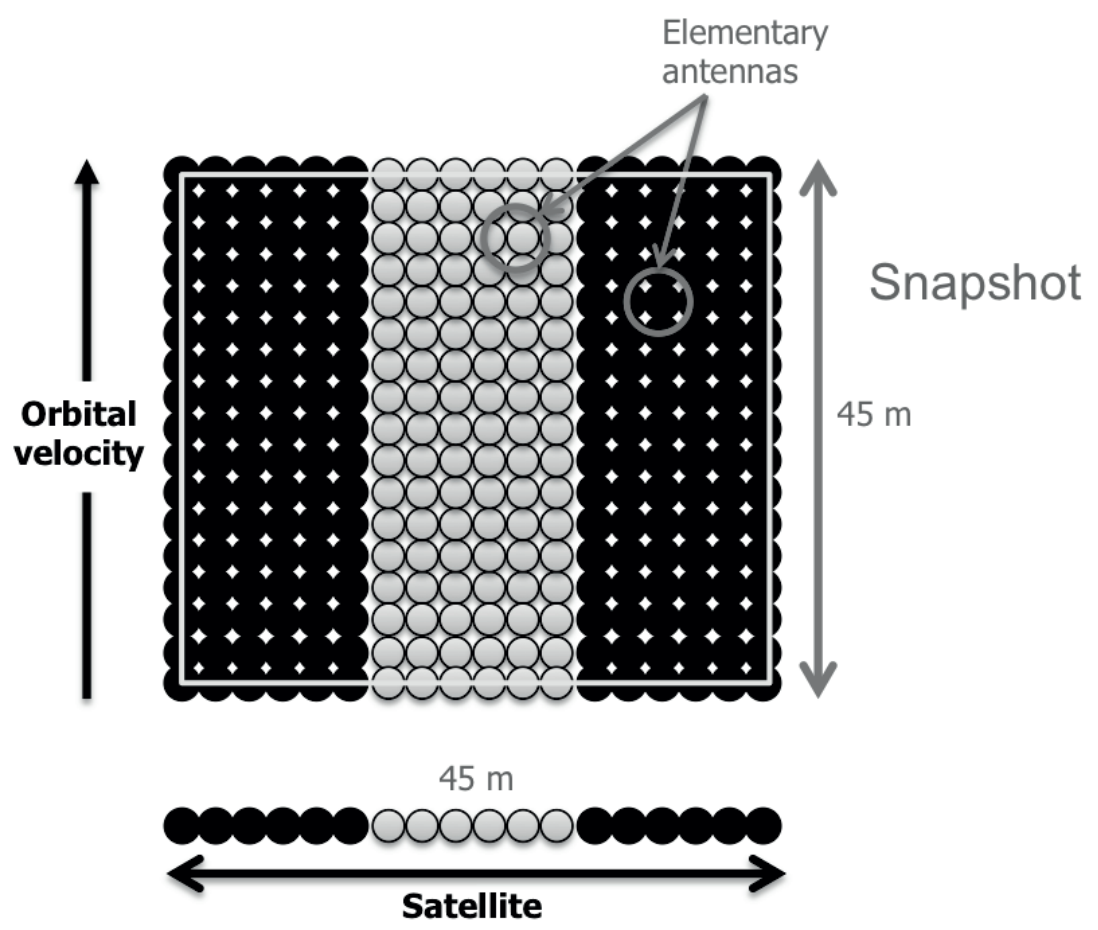

Fig. 3. Principle of spatio-temporal aperture synthesis: the phase differences are due to the difference in space and time between antennas.

\section{Spatio-temporal interferometry}

In order to improve the spatial resolution while maintaining roughly the same radiometric resolution, an original solution was proposed in Cabot et al. (2012) and Kerr et al. (2010). It consists in using observations made by a set of antennas at different times. The temporal coherence of a signal with spectral bandwidth $B$ is defined as:

$$
\tau=\frac{1}{B}
$$

With a fine filtering it is possible to select bandwidths as small as $100 \mathrm{~Hz}$, which results in coherence time of $0,01 \mathrm{~s}$. At the orbital speed, the satellite travels, within the coherence time, a distance of $75 \mathrm{~m}$, i.e. more than what it is required for a snapshot.

In this condition it is possible for the satellite to observe at two different times, two signals that are coherent with one another. Based on this principle, that was studied to depth in Braun 2011, SMOS-NEXT would be a 1-D interferometric radiometer, whose second dimension is given by the movement of the satellite on its orbit. In other words, signal acquired by the $i-t h$ elementary antenna at the 
instant $t_{0}$ would be correlated (also) to the acquisitions of the same antenna at later times $t_{0}+\Delta t$ (Fig. 3).

In this way the relation between spatial and radiometric resolution is less strict and it is possible to meet the requirements for both.

In fact with the elementary antennas along the arm and the satellite's motion, a set of both real and virtual antennas (i.e. real antennas at later times) is created, and while the spatial resolution is assured by the baselines in the two directions (along the satellite's arm and along the movement of the satellite), the radiometric resolution is only function of the real elementary antennas along the arm.

\section{Preliminary design}

SMOS-NEXT requires long physical baselines. But a satellite with three $40 \mathrm{~m}$ long arms disposed in a Y-shape is not a technologically realistic solution for the time being. Nevertheless it is possible to launch and deploy on orbit a single 40-45 m long arm. This is one of the two design options considered today, the other solution consisting in two satellites flying in formation.

Both solutions represent technical difficulties but the second one would imply a spatial resolution that depends on the relative distance between the satellites, which shortens at high latitudes, when the orbital planes cross. So we will consider only the first solution here on.

From the satellite's altitude, we can calculate its mean velocity:

$$
V_{\text {sat }}=\sqrt{\frac{\mu}{a}}
$$

where $\mu$ is the standard gravitational parameter equal to $398600 \mathrm{~km}^{3} \mathrm{~s}^{-2}$, and $a$ is the semi-major axis of the orbit. For a circular orbit and an altitude of $700 \mathrm{~km}$, $V_{\text {sat }}$ is roughly equal to $7500 \mathrm{~ms}^{-1}$.

The arm will be filled with elementary antennas spaced, as for SMOS, by 0,875 wavelengths. If the maximum redundancy configuration is chosen the total number of antennas will be roughly 250 .

Sampling frequency $\left(F_{s}\right)$ will be such that the spacing between real and virtual antennas $\left(\Delta s_{\text {antennas }}\right)$ is at least equal to the spacing between real antennas, that is:

$$
F_{s} \geq \frac{V_{\text {sat }}}{\Delta s_{\text {antennas }}}=\frac{7500 \mathrm{~ms}^{-1}}{0,875 \lambda}=\frac{7500 \mathrm{~ms}^{-1}}{0,875 \cdot 0,21 \mathrm{~m}} \approx 40 \mathrm{kHz} .
$$

A snapshot is then defined as the sequence of acquisitions that the satellite makes in a time interval corresponding to a displacement of about $45 \mathrm{~m}$. The time interval required for the satellite to cover this distance $(0,006 \mathrm{~s})$ must be lower than the coherence time for the chosen bandwidth $(0,01 \mathrm{~s})$. Each snapshot will then be composed by 240 or more acquisitions. 


\section{Cross-correlations between antennas}

The expression of an electromagnetic signal for a set of virtual and real antennas can be expressed in function of time and space as in:

$$
\frac{S_{0}(s, \nu)}{\rho(s)}=e^{-2 j \pi \nu\left(t-\frac{\rho(s, t)}{c}\right)}
$$

where $S_{0}$ is the electromagnetic signal, $\nu$ is the signal's frequency, $\rho(s, t)$ is the source-antenna distance, $t$ is the time and $s$ indicates the source's position.

Under the assumption that sources are spatially incoherent the cross-correlations between the two electromagnetic fields at the antennas' positions can be written as

$$
\left\langle S_{0}(s, \nu) S_{0}^{*}(s, \nu)\right\rangle \frac{e^{-2 j \pi \nu\left(t_{1}-\frac{\rho_{1}\left(s, t_{1}\right)}{c}\right)}}{\rho_{1}(s)} \frac{e^{-2 j \pi \nu\left(t_{2}-\frac{\rho_{2}\left(s, t_{2}\right)}{c}\right)}}{\rho_{2}(s)}
$$

where indexes 1 and 2 indicate the two antennas.

Even though the source-antenna distance varies with time due to Earth's oblateness and orbital ellipticity we can consider it independent from time during a snapshot.

If we consider the case in which 1 and 2 represent the same antenna at different times, then we have:

$$
\begin{array}{r}
\left\langle S_{0}(s, \nu) S_{0}^{*}(s, \nu)\right\rangle \frac{e^{-2 j \pi \nu\left(t_{1}-\frac{\rho_{1}\left(s, t_{1}\right)}{c}\right)}}{\rho_{1}(s)} \frac{e^{-2 j \pi \nu\left(t_{1}+\Delta t-\frac{\rho_{2}\left(s, t_{1}+\Delta t\right)}{c}\right)}}{\rho_{2}(s)} \\
\left\langle S_{0}(s, \nu) S_{0}^{*}(s, \nu)\right\rangle \frac{e^{-2 j \pi \nu\left(t_{1}-\frac{\rho_{1}\left(s, t_{1}\right)}{c}\right)}}{\rho_{1}(s)} \frac{e^{-2 j \pi \nu\left(t_{1}-\frac{\rho_{2}\left(s, t_{1}+\Delta t\right)}{c}\right)}}{\rho_{2}(s)} e^{2 j \pi \nu \Delta t .}
\end{array}
$$

We have then obtained the expression of the van Cittert-Zernike theorem multiplied by an exponential term.

In fact the term $\left\langle S_{0}(s, \nu) S_{0}^{*}(s, \nu)\right\rangle$ is simply the intensity of the electromagnetic radiation of the source, noted $T_{B}$, and by applying the far field approximation and the quasi monochromatic approximation, the product of the phase terms can be expressed as a function of the direction cosines $(\xi, \eta)$ as follows:

$$
\frac{e^{-2 j \pi \nu\left(t_{1}-\frac{\rho_{1}\left(s, t_{1}\right)}{c}\right)} \rho_{1}(s) e^{-2 j \pi \nu\left(t_{1}-\frac{\rho_{2}\left(s, t_{1}+\Delta t\right)}{c}\right)} \rho_{2}(s)}{\rho_{1}(s) \rho_{2}(s)} \simeq e^{-2 j \pi \nu \frac{\left(d_{1} \xi+d_{2} \eta\right)}{c}} .
$$

Integrating over the observation area we have:

$$
\begin{aligned}
V & =\iint_{\xi^{2}+\eta^{2}<1} \frac{T_{B}(\xi, \eta)}{\sqrt{1-\xi^{2}-\eta^{2}}} e^{-2 j \pi \nu \frac{\left(d_{1} \xi+d_{2} \eta\right)}{c}} e^{2 j \pi \nu \Delta t} \mathrm{~d} \xi \mathrm{d} \eta= \\
& =e^{2 j \pi \nu \Delta t} \iint_{\xi^{2}+\eta^{2}<1} \frac{T_{B}(\xi, \eta)}{\sqrt{1-\xi^{2}-\eta^{2}}} e^{-2 j \pi \nu \frac{\left(d_{1} \xi+d_{2} \eta\right)}{c}} \mathrm{~d} \xi \mathrm{d} \eta
\end{aligned}
$$


Aside from the term $e^{2 j \pi \nu \Delta t}$, in this expression the visibility $(V)$, corresponding to the cross-correlation between antennas, is expressed as the two-dimensional Fourier transform of the so-called modified brightness temperature map of the source, which is the brightness temperature divided by the obliquity factor $\sqrt{1-\xi^{2}-\eta^{2}}$ (Camps et al. 1998).

So far we only considered the central frequency of the signal. The integration of (6.7) on the filter's bandwidth $(B)$ can be written dropping the double integral since it is independent from frequency.

$$
\int_{\nu-\mathrm{B} / 2}^{\nu+\mathrm{B} / 2} e^{2 j \pi \nu \Delta t}=e^{2 j \pi \nu \Delta t} \operatorname{sinc}\left(B \frac{u \xi+v \eta}{\nu}\right)
$$

where

$$
\operatorname{sinc}\left(B \frac{u \xi+v \eta}{\nu}\right)=\tilde{r}(\xi, \eta)
$$

is called the Fringe Washing Function. By integrating 6.8 and 6.9 in 6.7 we obtain

$$
=e^{2 j \pi \nu \Delta t} \iint_{\xi^{2}+\eta^{2}<1} \frac{T_{B}}{\sqrt{1-\xi^{2}-\eta^{2}}} \tilde{r} e^{-2 j \pi \nu \frac{\left(d_{1} \xi+d_{2} \eta\right)}{c}} \mathrm{~d} \xi \mathrm{d} \eta .
$$

As soon as we consider real antennas, their radiation patterns $(F(\xi, \eta))$ and their corresponding solid angles $(\Omega)$ must be taken into account. In the case under study (same antenna, different times) the final expression is then written as follows

$$
V=e^{2 j \pi \nu \Delta t} \iint_{\xi^{2}+\eta^{2}<1} \frac{F(\xi, \eta)^{*} F(\xi, \eta)}{\Omega(\xi, \eta)} \frac{T_{B}(\xi, \eta)}{\sqrt{1-\xi^{2}-\eta^{2}}} \tilde{r} e^{-2 j \pi \nu \frac{\left(d_{1} \xi+d_{2} \eta\right)}{c}} \mathrm{~d} \xi \mathrm{d} \eta
$$

where $F^{*}(\xi, \eta)$ represents the complex conjugate of $F(\xi, \eta)$.

\section{Detemporalization}

Previously we made the choice of selecting a $100 \mathrm{~Hz}$ band. Even though possible, this solution represent technical difficulties, and in order to use wider range of the protected band several bandwidths of this amplitude would be needed, thus multiplying the quantity of information to be downlinked to the ground stations.

A different approach is therefore proposed. It consists in using larger bandwidths and applying a temporal shift to the signal received by one of the antennas.

This approach is called detemporalization.

This is implemented by multiplying by $e^{-2 j \pi \nu \Delta t}$ the phasor describing the electromagnetic field at time $t_{2}$ that appear in the precedent expression. Following the same development, we obtain hereafter the expression for the visibility function:

$$
V=\frac{1}{\Omega} \iint_{\xi^{2}+\eta^{2}<1} F^{*} F \frac{T_{B}}{\sqrt{1-\xi^{2}-\eta^{2}}} \tilde{r} e^{-2 j \pi \nu \frac{\left(d_{1} \xi+d_{2} \eta\right)}{c}} \mathrm{~d} \xi \mathrm{d} \eta
$$


That is the fundamental relationship between visibility and brightness temperature used for SMOS, before considering the effects of antenna radiation patterns, and before integration with respect to the frequency.

\section{Disaggregation}

This information on ground will then provide brightness temperature global maps, and using several observations the soil moisture can be retrieved. The spatial resolution of these maps will be, using the data explicated above:

$$
R_{s}=\frac{H \lambda}{d}<4 \mathrm{~km}
$$

This result represents a significant improvement with respect to the data available for the time being, but still it is not sufficient for integration with the hydrological models. In order to do so, this data needs to be downscaled further. Disaggregation methods allow downscaling of soil moisture microwave measurements, by making use of the knowledge of the evaporative fractions over specific areas, that are retrieved by optical, near-infrared or thermal infrared measurements (Merlin et al. 2008).

\section{Conclusions}

The objective of improving the spatial resolution of soil moisture and ocean salinity maps by an order of magnitude can be achieved with the use of a long baseline spatio-temporal interferometer.

The detemporalization technique was then introduced to ease the technical constraints of such instrument.

The resolution obtained is not yet sufficient for the implementation in hydrological models and in future weather models, in which the spatial resolution will be improved. Then disaggregation methods can then be used to downscale further space borne microwave soil moisture retrievals.

Theoretical studies have been conducted to study the principle of the spatiotemporal aperture synthesis, and experimental campaigns are going to be carried out in the near future.

\section{References}

Alcamo, J., Henrichs, T., \& Rösch, T., 2000, "World Water in 2025 - Global modeling and scenario analysis for the World Commission on Water for the 21st Century", Kassel World Water Series 2, Center for Environmental Systems Research (University of Kassel, Germany)

Anterrieu, E., \& Khazaal, A., 2011, "One Year Of RFI Detection And Quantification With L1a Signals Provided By SMOS Reference Radiometers", Geoscience and Remote Sensing Symposium (IGARSS) 2011 IEEE International, 2245

Bartalis, Z., Wagner, W., Naeimi, V., et al., 2007, Geophys. Res. Lett., 34 
Berger, M., Camps, A., Font, J., et al., 2002, "Measuring Ocean Salinity with ESA's SMOS Mission", ESA Bulletin, No. 111, 113

Braun, D., 2011, "Physical analysis of spatial and temporal correlations for SMOS-next"

Cabot, F., Kerr, Y.H., Anterrieu, E., et al., 2012, "SMOS-Next: New perspectives for Soil Moisture and Ocean Salinity from space", Microrad (Frascati, IT, 5-9 March 2012)

Camps, A., Corbella, I., Bara, J., \& Torres, F., 1998, IEEE Trans. Geosci. Remote Sensing, 36, 680

Dai, A., Trenberth, K.E., \& Qian, T., 2004, J. Hydrometeor., 5, 1117

Döll, P., Kaspar, F., \& Lehner, B., 2003, J. Hydrology, 270, 105

Entekhabi, D., Njoku, E.G., O'Neill, P.E., et al., 2010, Proc. IEEE, 98, 704

Grodsky, S.A., Reul, N., Lagerloef, G., et al., 2012, Geophys. Res. Lett., L20603

ITU Radio Regulations, 1996, Frequency Allocation National Bulletin, Official Bull. Span State, Aug. 9

Kaleschke, L., Tian-Kunze, X., Maass, N., Mäkynen, M., \& Drusch, M., 2012, Geophys. Res. Lett., L05501

Kerr, Y.H., Waldteufel, P., Wigneron, J.-P., et al., 2001, Geosc. Remote Sensing, IEEE Trans., 39, 1729

Kerr, Y.H., Rougé, B., Cabot, F., et al., 2010, "SMOS NEXT: The new generation", Microrad (Washington DC, USA, 1-4 March 2010)

LeVine, D.M., Lagerloef, G.S.E., Colomb, F.R., Yueh, S.H., \& Pellerano, F.A., 2007, Geosc. Remote Sensing, IEEE Trans., 45, 2040

Merlin, O., Chehbouni, A., Walker, J.P., Panciera, R., \& Kerr, Y.H., 2008, Geosc. Remote Sensing, IEEE Trans., 46, 3

Njoku, E.G., Jackson, T.J., Chan, T.K., \& Nghiem, S.V., 2003, Geosc. Remote Sensing, IEEE Trans., 41, 215

Robock, A., Vinnikov, K.Y., Srinivasan, G., et al., 2000, Bull. Amer. Meteor. Soc., 81, 1281

Roemmich, D., \& Owens, W.B., 2000, Oceanography, 13, 45

Wigneron, J.P., Chanzy, A., Waldteufel, P., et al., 2000, "Retrieval capabilities of LBand 2-D interferometric radiometry over land surfaces (SMOS Mission)" (VSP, The Netherlands) 